\title{
Uma Revisão das Diferentes Abordagens Computacionais para Detecção de Estilos de Aprendizagem de Estudantes em Sistemas para Educação a Distância
}

\section{A Review of Different Computational Approaches for Detecting of Student Learning Styles in Systems for Distance Education}

\author{
ARTHUR MACHADO FRANÇA DE ALMEIDA \\ Instituto Federal do Norte de Minas \\ Gerais (IFNMG)
LUCIANA PEREIRA DE ASSIS
Universidade Federal dos Vales do Jequitinhonha e Mucuri (UFVJM) \\ ALESSANDRO VIVAS ANDRADE \\ Universidade Federal dos Vales do \\ Jequitinhonha e Mucuri (UFVJM)
}

\begin{abstract}
Resumo: Com a evolução da Educação a Distância nos últimos anos, muito se tem estudado sobre a importância de se considerar estilos de aprendizagem no processo de ensino a distância. No entanto, a identificação dos estilos de aprendizagem de um estudante em um ambiente EaD não é uma tarefa trivial. Este artigo busca realizar uma revisão sobre as diferentes abordagens computacionais para detecção de estilos de aprendizagem presentes na literatura. As abordagens computacionais aqui apresentadas são baseadas em técnicas da Inteligência Artificial capazes de realizar a deteç̧ão dos estilos de aprendizagem de forma automática a partir do comportamento do aluno em um ambiente virtual de aprendizagem. No total, foram selecionados 26 artigos, dos quais pode-se analisar 15 abordagens diferentes para detecção de estilos de aprendizagem. Dentre as abordagens, a mais utilizada nos trabalhos selecionados foram as Redes Bayesianas. Além disso, percebeu-se que as pesquisas relacionadas a detecção automática de estilos de aprendizagem, mesmo após as críticas recentes a teoria, continuam sendo desenvolvidas e aperfeiçoadas.
\end{abstract}

Palavras-chave: Estilos de Aprendizagem. Educação a Distância. Inteligência Artificial. Abstract: With the evolution of Distance Education in recent years, much has been studied about
the importance of considering learning styles in the distance learning process. However, identifying
a student's learning styles in an EaD environment is not a trivial task. This article seeks to review
the different computational approaches for detecting learning styles present in the literature. The
computational approaches presented here are based on Artificial Intelligence techniques capable of 
automatically detecting learning styles from the student's behavior in a virtual learning environment. In total, 26 articles were selected, of which 15 different approaches for detecting learning styles can be analyzed. Among the approaches, the most used in the selected works were the Bayesian Networks. In addition, it was noticed that research related to the automatic detection of learning styles, even after recent criticisms of the theory, continues to be developed and improved.

Keywords: Learning Styles. Distance Education. Artificial Intelligence.

ALMEIDA, Arthur Machado França; ASSIS, Luciana Pereira; ANDRADE, Alessandro Vivas. Uma Revisão das Diferentes Abordagens Computacionais para 'Detecçãó de Estilos de Áprendizagem de Estudantes em Sistemas para Educação a Distância. Informática na Educação: teoria \& prática, Porto Alegre, v. 23, n. 1, p. 95-113, jan./abr. 2020.

\section{Introdução}

A Educação a Distância (EaD) tem crescido mundialmente nos últimos anos. No Brasil, a realidade não é diferente, visto que a EaD é incentivada pela Lei de Diretrizes e Bases da Educação (BRASIL, 1996). Segundo dados do Instituto Nacional de Estudos e Pesquisas Educacionais Anísio Teixeira (INEP), o número de cursos de graduação a distância no Brasil cresceu 571\% entre 2003 e 2006. Entre 2011 e 2014, esse número cresceu 213\% (BITTENCOURT; MERCADO, 2014).

Com o advento da Internet, diversas ferramentas para uso coletivo surgiram. Na área da educação, os sistemas de gestão de aprendizagem (Learning Management Systems - LMS) vieram revolucionar a Educação a Distância, disponibilizando diversos recursos que dão suporte ao processo de ensino-aprendizagem, auxiliando no seu planejamento, implantação e avaliação.

Segundo Dorça (2012), os sistemas de gestão de aprendizagem fornecem grande apoio aos professores, auxiliando-os na autoria e realização de cursos online. Porém, esses sistemas não consideram as diferenças individuais dos estudantes, fornecendo um suporte limitado aos usuários (GRAF et al., 2010), pois o mesmo conteúdo e estratégia pedagógica são utilizados para todos os discentes.

Diferentes estudantes possuem diferentes preferências no aprendizado. Cada pessoa possui uma forma diferente de melhor compreender alguma situação. De acordo com Silva (2006), cada pessoa tem uma forma única para perceber e adquirir conhecimento a partir do processo ensino-aprendizagem. Dessa forma, os estilos de aprendizagem (EAs) representam um conjunto de habilidades e experiências que estão diretamente relacionadas com o estado cognitivo de cada pessoa (SILVA, 2006). O EA refere-se à forma como cada indivíduo assimila e processa as informações que recebe (SOARES; SOARES, 2019).

Os EAs representam um papel importante no processo de aprendizagem. Através deles é possível conhecer a forma como os alunos aprendem e, assim, selecionar as melhores estratégias de ensino (ZAPALSKA; BROZIK, 2006). Silva (2012) afirma que compreender essas diferenças relacionadas a estilos de aprendizagem e estilos cognitivos pode ser o primeiro passo que as instituições educacionais devam dar para alcançar uma aprendizagem eficaz. Ainda, de acordo com Barros (2008), a teoria dos estilos de aprendizagem é flexível e se apoia 
nas diferenças individuais, contribuindo para a construção do processo de ensino e aprendizagem na perspectiva de uso das tecnologias.

Dessa forma, muito se tem estudado sobre a detecção automática de estilos de aprendizagem nos Sistemas para Educação a Distância. Nos últimos anos diversos trabalhos foram desenvolvidos, apresentando novas técnicas computacionais para a solução do problema de detecção de EA. Este artigo busca fazer uma revisão dos principais métodos computacionais que vêm sendo desenvolvidos para realizar a detecção de estilos de aprendizagem nos Sistemas de Gestão de Aprendizagem.

\section{Educação a Distância e Estilos de Aprendizagem}

A EaD é uma modalidade de ensino caracterizada pela separação física entre aluno e professor (RAMOS, 2010). Ela possui elementos interativos que englobam os processos de ensino, aprendizagem, comunicação e controle (MOORE; KEARSLEY, 2011). Devido à flexibilidade e os preços acessíveis, o número de pessoas que buscam os cursos EaD no Brasil têm se multiplicado (NETTO; SOUZA, 2019). Hoje ela é considerada uma importante ferramenta para difusão do conhecimento e democratização da informação (SILVA, 2019).

Com o rápido crescimento da internet e o surgimento do e-learning, os sistemas de gestão de aprendizagem (LMS) surgiram para auxiliar a EaD. Os LMS são sistemas especializados com tecnologias para o ensino e aprendizagem baseados na Internet e na Web, que visam fornecer educação e treinamento seguindo o paradigma da educação a distância (AVGERIOU et al., 2003).

Os LMS possibilitam que os professores desenvolvam o material instrucional, os exercícios e testes, e facilitam a comunicação com os alunos. Para os alunos, um LMS deve possibilitar o acesso ao material disponibilizado e fornecer ferramentas de comunicação com seus colegas e com os professores e monitores (NETO, 2006).

Apesar dos LMS fornecerem grande apoio aos professores e alunos, esses sistemas não consideram as diferenças individuais dos estudantes e o mesmo conteúdo é apresentado para todos os usuários. Para Puga (2008), esses sistemas auxiliam na organização e controle das atividades programadas em um curso, mas não auxiliam o professor na preparação ou na escolha do conteúdo mais adequado a ser apresentado ao aluno. Diante desse cenário, a adaptatividade desses sistemas a diferentes estudantes tem sido frequentemente discutida.

Segundo Dorça (2012), muito se tem discutido sobre a modelagem do estudante baseada em estilos de aprendizagem. Dessa forma, as preferências psicológicas são armazenadas em bases de dados e então utilizadas no fornecimento de estratégias de ensino personalizadas (BROWN et al., 2006). Essa base de dados é denominada Modelo do Estudante (ME), que é destinada a armazenar as características dos estudantes e que são utilizadas para que o sistema possa realizar a adaptação (PUGA, 2008).

Basicamente, estilos de aprendizagem são características básicas de como se aprende uma nova informação, e não são perceptíveis de forma consciente pelos estudantes (REID, 1998). Felder e Brent (2005) definem os estilos de aprendizagem como características cognitivas que servem como um indicador de como o estudante percebe, interage e responde aos ambientes de aprendizagem. 
Ao longo do tempo, diversos modelos foram desenvolvidos para a estruturação e descrição de Estilos de Aprendizagem. Dentre os modelos desenvolvidos, pode-se destacar: Kolb et. al (1984), Honey e Mumford (1992), Pask (1976) e Felder et. al (1988). O Felder-Silverman Learning Style Model - FSLSM (FELDER et. al, 1988) é o modelo que vem sendo utilizado pelas abordagens computacionais, principalmente devido a sua simplicidade na representação: é composto por apenas quatro dimensões.

As dimensões do FSLSM são divididas em subdimensões e se caracterizam da seguinte forma (DORÇA, 2012):

- Percepção: essa dimensão refere-se ao tipo de informação que o estudante prefere receber. Subdivide-se em Sensitivo, com preferência por fatos, datas, dados experimentais, exemplos; e Intuitivo, com preferência por teorias, definições, modelos matemáticos.

- Entrada: essa dimensão refere-se ao tipo de informação que é mais efetivamente percebida. Subdivide-se em Visual, com inclinação por figuras, diagramas, gráficos, filmes; e Verbal, com inclinação por explicação escrita ou falada.

- Processamento: essa dimensão refere-se à preferência do estudante sobre o processamento da informação. Subdivide-se em Ativo, com preferência por experimentação ativa, discussões, trabalho em grupo; e Reflexivo, com preferência por trabalho individual, introspecção, reflexão, teoria.

- Organização: essa dimensão refere-se à preferência de progressão do estudante dentro do curso. Subdivide-se em Sequencial, com inclinação por uma progressão lógica e linear no conteúdo; e Global, com inclinação por uma visão geral do todo, aprendendo aleatoriamente.

Os estudantes apresentam tendências de preferências em cada uma das quatro dimensões (FELDER; SPURLIN, 2005). As dimensões definem as formas de receber, processar, perceber e organizar a informação (DORÇA, 2012). Uma característica do FSLSM é que suas dimensões não são estáticas, ou seja, um estudante pode tender de um Estilo de Aprendizagem para outro, dentro de uma mesma dimensão.

Para Barros (2008), o espaço virtual apresenta possibilidades de aprendizagem diferenciadas das tradicionais do ensino presencial e os estilos de aprendizagem apresentam características que podem ser identificadas dentro do espaço virtual. Dessa forma, os estilos de uso do espaço virtual aliados aos estilos de aprendizagem podem auxiliar na identificação do perfil de como um indivíduo aprende, permitindo um direcionamento das ações pedagógicas (MIRANDA, 2012). Os estilos de uso do espaço virtual, embora sejam importantes, não são foco da presente pesquisa e por isso seus conceitos não serão aprofundados.

\subsection{Críticas aos Estilos de Aprendizagem}

Embora exista na literatura um rico estudo sobre os EAs e uma variedade de modelos que buscam descrevê-los, algumas pesquisas recentes têm questionado a teoria. Essas pesquisas levantam possíveis falhas na teoria e modelos de EA.

Uma das críticas está relacionada ao aspecto determinístico que os EAs supostamente 
apresentam. Kirschner e Merriënboer (2013) criticam a concepção de EA como uma característica fixa do indivíduo, onde o EA é estático e não pode variar ao longo do tempo. Crítica semelhante apresenta Pashler et al. (2008), ao constatarem que essa definição de um EA fixo pode levar o indivíduo a entender que não consegue aprender de determinadas formas, que não sejam aquelas de sua preferência.

Outra crítica aos EAs está relacionada às suas formas de detecção. Rawson; Stahovich; Mayer (2017) alegam que a identificação de EA por meio de questionários muitas vezes não condiz com a realidade, visto que existe uma baixa correlação entre as respostas e o que é, de fato, verdade. Veenman; Prins; Verheij (2003) criticam o uso de questionários psicométricos para realizar a detecção dos EAs, visto que os estudantes não conseguem definir claramente a forma que melhor aprendem. Graf, Lin et al. (2007) abordam a imprecisão e grau de incerteza associados a este tipo de questionário para autoavaliação de EA. Outras críticas também são apresentadas nos trabalhos de Kirshner (2017) e Nancekivell; Shah; Gelman (2019).

Apesar das críticas, pesquisas recentes têm explorado a utilização de EAs nos Sistemas de Aprendizagem. Trabalhos como Dorça (2012), Sena et al. (2016), Rodrigues et al. (2016), Falci et al. (2017), Salazar et al. (2017), Ribeiro et al. (2017) e Silva et al. (2018) apresentam abordagens para detecção de EAs de forma automática, baseadas em técnicas de Inteligência Artificial e Aprendizagem de Máquina. As abordagens para detecção de EA de forma automática afastam uma das principais críticas à teoria, já que dispensam o uso de questionários para autoavaliação dos estudantes.

Todos esses trabalhos utilizam ainda o FSLSM como modelo para descrição de EA. O FSLSM é um modelo dinâmico e probabilístico, onde os estudantes possuem tendências de preferências em cada dimensão (DORÇA, 2012). Sendo assim, o estudante possui uma probabilidade de pertencer a uma determinada dimensão de EA, mas possui também probabilidades menores de pertencer a outros estilos (DORÇA, 2012). Assim, o uso do FSLSM como modelo uma outra crítica à teoria, de que os EAs são tratados de forma determinística.

Ressalta-se, ainda, que as críticas aos EAs apresentadas neste trabalho, retiradas de Kirschner e Merriënboer (2013), Pashler et al. (2008), Rawson; Stahovich; Mayer (2017) e Veenman; Prins; Verheij (2003), fazem mais sentido quando aplicadas ao ensino presencial, já que no ensino na modalidade EaD existe o uso de ferramentas computacionais, que facilitam a detecção automática dos EAs e a classificação de acordo com um modelo como o FSLSM.

\section{Detecção de Estilos de Aprendizagem}

Uma das formas mais utilizadas para realizar a detecção de EA é a automática. Nas abordagens automáticas, de acordo com o comportamento e as ações tomadas pelo estudante dentro do sistema, o ambiente consegue detectar e atualizar o estilo de aprendizagem do estudante automaticamente (BRUSILOVSKY, 1996).

Com o decorrer do tempo, diversas abordagens para detecção e correção de estilos de aprendizagem foram desenvolvidas. Algumas delas são abordagens colaborativas que utilizam questionários. Outras são abordagens computacionais que utilizam técnicas de Aprendizado de Máquina, Inteligência Computacional e Inteligência Artificial. 
Na revisão bibliográfica realizada nesse trabalho, foram selecionados 26 trabalhos sobre detecção automática de estilos de aprendizagem. Eles possuem em comum o fato de utilizarem técnicas de Inteligência Artificial para identificar os EAs. Foram considerados os trabalhos clássicos de detecção automática de estilos de aprendizagem, ou seja, aqueles realizados por pesquisadores que já publicaram muitos trabalhos na área e possuem pelo menos 5 citações; além de trabalhos importantes desenvolvidos por pesquisadores brasileiros (as) e publicados em eventos e periódicos nacionais. Os buscadores utilizados para a pesquisa foram a plataforma Periódicos Capes e o Google Acadêmico.

Nos próximos tópicos são descritas as principais técnicas da Inteligência Artificial para detecção de estilos de aprendizagem desenvolvidas nos últimos anos.

\subsection{Redes Bayesianas}

As Redes Bayesianas são uma representação compacta e expressiva de relações incertas entre parâmetros de um domínio (GARCIA et al., 2007). São estruturas capazes de modelar situações que apresentam algum grau de incerteza e não são determinísticas.

Dessa forma, as Redes Bayesianas se adéquam muito bem na detecção de estilos de aprendizagem, se considerarmos que esse é um problema não determinístico e que apresenta determinado grau de incerteza. De acordo com Al-Azawei e Badii (2014), com a técnica de Redes Bayesianas é possível criar uma rede entre os padrões de comportamento do estudante e as dimensões do modelo de estilo de aprendizagem utilizado.

Vários autores já desenvolveram pesquisas relacionadas à detecção dos estilos de aprendizagem utilizando a abordagem de Redes Bayesianas. Podemos citar, por exemplo, os trabalhos de Garcia et al. (2005), Garcia et al. (2007), Garcia et al. (2008), Botsios et al. (2008), Essaid; El Bachari; El Adnani (2011) e Salazar et al. (2017).

O trabalho de Garcia et al. (2007) foi um dos primeiros a relacionar Redes Bayesianas e o FSLSM. Garcia et al. (2007) utiliza o Teorema de Bayes para inferir as características do estudante após observar seu comportamento durante o processo de aprendizagem.

Para avaliar sua abordagem, Garcia et al. (2007) utilizou 27 estudantes de um curso de Inteligência Artificial em um Ambiente Virtual de Aprendizagem. Os resultados foram comparados com resultados obtidos pelo ILS, proposto por Felder. Foi verificado um grande índice de acerto entre as probabilidades inferidas pela Rede Bayesiana e o resultado obtido pelo questionário ILS.

Dessa forma, Garcia et al. (2007) conseguiu comprovar com sua pesquisa a eficiência e viabilidade do uso de Redes Bayesianas para detecção e correção automática dos estilos de aprendizagem utilizando o FSLSM em ambientes virtuais de aprendizagem reais.

Já em Amir et al. (2016) é proposto uma abordagem para detecção de estilos de aprendizagem que utiliza Máquinas de Vetores de Suporte. Uma Máquina de Vetores de Suporte (Support Vector Machine - SVM) é uma técnica da área da Inteligência Computacional, baseada na Teoria de Aprendizado Estatístico (GONG; WANG, 2011). Uma SVM é um classificador binário, pois possui como entrada um conjunto de dados e infere, para cada entrada, qual das duas classes a entrada faz parte. 
Amir et al. (2016) analisaram dados de duzentos alunos através dos logs de arquivos de um sistema de gestão de aprendizagem. Utilizaram, então, o SVM para inferir os estilos de aprendizagem dos estudantes. Os resultados foram comparados com resultados obtidos utilizando o classificador Naive Bayes. Os resultados da SVM se mostraram superiores aos resultados utilizando Naive Bayes, o que comprova a viabilidade da proposta (AMIR et al., 2016).

\subsection{Redes Neurais Artificiais}

As Redes Neurais Artificiais são modelos computacionais baseados na estrutura dos neurônios do cérebro humano e tem como objetivo reproduzir a maneira como o sistema nervoso humano trabalha (VILLAVERDE et al., 2006). O principal elemento da rede é o neurônio artificial, que está conectado a outros neurônios, e estes formam uma rede de neurônios. Por meio desta rede, os sinais são transmitidos e geram as sinapses, responsáveis pelo aprendizado da rede (VILLAVERDE et al., 2006).

Alguns autores já desenvolveram pesquisas relacionadas à detecção e correção de estilos de aprendizagem utilizando a abordagem de Redes Neurais Artificiais. Podemos citar Villaverde et al. (2006), Cabada et al. (2011), Lo et al. (2012) e Bernard et al. (2015).

Lo et al. (2012) propõe um sistema de aprendizagem baseado na web que possui dois módulos: o modelo do estudante e o modelo adaptativo. O modelo do estudante, após coletar informações sobre o comportamento do mesmo durante as sessões de aprendizagem, faz uso de uma rede neural do tipo multicamada feed-forward para classificar o comportamento do aluno.

A validação da abordagem foi feita com estudantes reais. Os resultados mostraram que 0 treinamento da rede neural foi apropriado e o ambiente conseguiu inferir o estilo cognitivo do estudante com um alto grau de precisão (LO et al., 2012). Os autores consideraram, por fim, que o uso da rede neural multicamada possui potencial para poder ser utilizado em um ambiente virtual de aprendizagem.

Embora a utilização de redes neurais para deteç̧ão dos estilos de aprendizagem dos estudantes tenha se mostrado promissora, Al-Azawei e Badii (2014) ressaltam que essa abordagem deve ser melhor avaliada, pois apresenta um elevado custo computacional e grande complexidade.

\section{3 Árvores de Decisão}

De acordo com Russell e Norvig (1995), uma Árvore de Decisão é uma estrutura computacional utilizada para aprendizado de máquina e tomada de decisão. As árvores utilizam uma situação problema (conjunto de atributos) como entrada e geram uma hipótese como saída. Esse método é bastante utilizado por apresentar estrutura simples, porém poderosa.

O problema de deteç̧ão e correção automática dos estilos de aprendizagem já foi modelado por alguns autores utilizando a estrutura de Árvore de Decisão. Cha et al. (2006) e Ozpolat e Akar (2009) são exemplos de pesquisadores que utilizaram essa abordagem para identificação dos estilos de aprendizagem. 
Ozpolat e Akar (2009) utilizam uma estrutura de árvore combinada com o algoritmo NBTree e o classificador Binary Relevance para modelar os estilos de aprendizagem, baseados no FSLSM. Nessa abordagem, o estudante interage com o ambiente virtual de aprendizagem, que oferece objetos de aprendizagem genéricos ao estudante. De acordo com as opções do estudante pelos objetos, as mesmas são agrupadas e organizadas na árvore de decisão (OZPOLAT e AKAR, 2009).

A partir da geração da árvore, utiliza-se o algoritmo NBTree para inferir o estilo de aprendizagem do estudante. Assim, os estudantes são classificados de acordo com seus interesses e, conforme o resultado da classificação é identificado os estilos de aprendizagem (OZPOLAT e AKAR, 2009).

Já a abordagem proposta por Cha et al. (2006) utiliza Árvores de Decisão combinadas com Modelos Ocultos de Markov. Através da interação do estudante com o ambiente virtual de ensino, o sistema coleta suas preferências individuais e as armazena em uma estrutura de Árvore de Decisão. A detecção dos estilos de aprendizagem é realizada utilizando o Modelo Oculto de Markov, um método estatístico que utiliza a sequência de observações e as probabilidades para inferir os resultados.

\subsection{Algoritmos Genéticos}

Algoritmos Genéticos são heurísticas baseadas na Teoria da Evolução de Charles Darwin. Com base nessa técnica, uma população de soluções candidatas a um problema de otimização evolui para melhores soluções, através dos princípios naturais de herança, mutação, seleção natural e recombinação (YANNIBELLI et al., 2006).

Algumas abordagens para identificação de estilos de aprendizagem utilizando Algoritmos Genéticos já foram propostas. Chang et al. (2009) propõe um método para classificar e identificar estilos de aprendizagem de estudantes. O autor utiliza o $\mathrm{K}$-Nearest Neighbor como classificador, juntamente com um Algoritmo Genético.

Yannibelli et al. (2006) utiliza um Algoritmo Genético que busca identificar as ações do aluno ao frequentar uma disciplina. Assim, as ações observadas são consideradas como as preferências do estudante e então são mapeadas para o FSLSM. No seu algoritmo, Yannibelli et al. (2006) define a população inicial de cromossomos, de forma que cada cromossomo representa uma combinação de ações. Cada cromossomo dessa população é avaliado de acordo com as ações feitas pelo aluno. Uma nova população é obtida por meio de técnicas de seleção, crossover e mutação (YANNIBELLI et al., 2006).

Yannibelli et al. (2006) realizou testes com uma base de dez estudantes e seu algoritmo mostrou um alto índice de acerto nos estilos de aprendizagem. A taxa de acerto na detecção foi de $80 \%$ para a dimensão Processamento, $100 \%$ para a dimensão Percepção e $100 \%$ para a dimensão Organização. Yannibelli et al. (2006) não considerou no seu algoritmo a dimensão Entrada do FSLSM.

\subsection{Aprendizagem por Reforço e Cadeias de Markov}

A Aprendizagem por Reforço é uma técnica da Inteligência Artificial que utiliza recompensas para que o agente possa aprender (RUSSEL; NORVIG, 1995). Através da interação do agente 
com o ambiente, o mesmo consegue aprender, de acordo com as recompensas que Ihe são oferecidas.

De acordo com Dorça (2012), a Aprendizagem por Reforço é geralmente utilizada quando não se consegue obter exemplos de qual o comportamento correto que o agente deve ter em determinadas situações, ou quando o agente está em um ambiente desconhecido. Assim, logo após realizar alguma ação, o agente toma conhecimento do estado alcançado e consegue obter experiência sobre o ganho de suas ações em determinado estado (DORÇA, 2012).

Sendo assim, a Aprendizagem por Reforço é um bom método para ser utilizado em situações onde não se possui uma base de conhecimento capaz de informar os objetivos e ações corretas, como é o caso da detecção automática e dinâmica de estilos de aprendizagem (DORÇA, 2012).

Dorça (2012) apresenta uma abordagem que utiliza Aprendizagem por Reforço, onde o processo de atualização do Modelo do Estudante é feito a partir do desempenho dos estudantes. O desempenho do estudante, por sua vez, é aferido utilizando-se um Processo Estocástico para Simulação do Desempenho do Estudante - PESDE, metodologia criada pelo autor.

O Modelo do Estudante é modelado com uma cadeia de Markov para cada dimensão do FSLSM, e é formado por uma combinação de objetivos de aprendizagem, estilos de aprendizagem e nível cognitivo.

A abordagem de Dorça (2012) utiliza o algoritmo Q-learning para, de acordo com o resultado do desempenho do estudante calculado pelo PESDE, realizar a retroalimentação e atualização do modelo de estudante, representado pelas cadeias de Markov.

Para realizar os testes e validar sua abordagem, Dorça (2012) utilizou a metodologia de simulação computacional. Os resultados obtidos foram satisfatórios, pois foi detectado um alto grau de coesão entre o modelo do estudante probabilístico e o modelo do estudante real, com um baixo custo computacional.

\subsection{Dynamic Scripting}

O Dynamic Scripting é uma técnica de Aprendizagem por Reforço online. Dessa forma, o aprendizado é concomitante ao próprio uso do sistema (SPRONCK, 2005). O Dynamic Scripting é capaz de aprender a partir de poucas tentativas por meio de scripts tradicionais da IA, que limitam o tamanho do espaço de estados (SPRONCK, 2005). Já a Aprendizagem por Reforço tradicional demanda grande quantidade de tentativas para convergência e por isso não são eficientes para a aprendizagem online (RABIN, 2002).

Nos trabalhos de Silva et al. (2017) e Silva et al. (2018), é apresentada uma nova forma de reforço no modelo proposto em Dorça (2012). A técnica é uma adaptação do Dynamic Scripting, denominada Adapted Dynamic Scripting (ADS). O ADS é composto por um conjunto de regras que contém condições, uma ação e um peso. As condições usam a nota do estudante e/ou a distância entre os estilos de aprendizagem. As ações consistem na aplicação de reforço positivo ou negativo no Estilo de Aprendizagem probabilístico. Já o peso determina a probabilidade de seleção de determinada regra. A base de regras do ADS é composta por 40 regras, de forma que para compor um script são necessárias 20 regras (SILVA et al., 2018). 
Os resultados obtidos pela abordagem se mostraram superiores ao apresentado em Dorça (2012). Para a comparação, foram apresentadas duas métricas: número de problemas de aprendizagem e nota média dos estudantes. Em relação ao número de problemas de aprendizagem, a abordagem ADS reduziu em aproximadamente $54 \%$ o número de problemas de aprendizagem para EAs estáticos e 35\% para EAs dinâmicos (SILVA et al., 2018). Na métrica nota média dos estudantes, a média de notas foi aproximadamente $6,06 \%$ maior que as notas obtidas na abordagem proposta em Dorça (2012) (SILVA et al., 2018).

\subsection{Mineração de Dados Educacionais}

A Mineração de Dados tem como objetivo utilizar técnicas para descoberta de conhecimento em bases de dados. Recentemente muito se tem discutido sobre a Mineração de Dados Educacionais, que consiste em aplicar métodos da Mineração de Dados para a descoberta de conhecimento em bases de dados de ambientes educacionais (BAKER et al., 2011).

Senechal (2013) afirma que essa abordagem utiliza informações da interação do aluno com o ambiente virtual, armazenadas nos arquivos de logs, para buscar padrões e inferir os estilos de aprendizagem dos estudantes.

De acordo com Baker et al. (2011), uma das principais linhas de pesquisa da Mineração de Dados Educacionais é o desenvolvimento de métodos eficazes para dar suporte ao aluno que utiliza ambientes virtuais de aprendizagem. Ainda, segundo o autor, alguns Sistemas Tutores Inteligentes na Europa e EUA já utilizam técnicas de Mineração de Dados Educacionais para proporcionar uma aprendizagem mais personalizada e de melhor qualidade.

Existe ainda a possibilidade de utilizar os dados coletados como entrada para outros algoritmos da Inteligência Artificial que possam realizar a inferência dos estilos de aprendizagem (AL-AZAWEI; BADII, 2014). Assim, os dados podem servir de insumo para técnicas como as Redes Bayesianas, Redes Neurais Artificiais, entre outros.

Esse é o caso do trabalho de Rajper et al. (2016), que utiliza técnicas de mineração de dados e Redes Bayesianas para inferir os estilos de aprendizagem. A pesquisa foi realizada com 863 estudantes matriculados em cursos de Ciência da Computação. Para validação da pesquisa, os resultados foram comparados com resultados do questionário KLSI, utilizado para classificar os estudantes de acordo com o modelo do Kolb's Learning Style Model. Por fim, o autor avalia que os resultados obtidos podem melhorar no futuro com o aprimoramento da técnica (RAJPER et al., 2016).

\subsection{Lógica Fuzzy}

A Lógica Fuzzy é baseada na teoria dos conjuntos fuzzy e se difere dos sistemas lógicos tradicionais devido a suas características e detalhes (GOMIDE et al., 1995). É a lógica onde os modelos de raciocínio são aproximados, e não exatos. Na Lógica Fuzzy, o valor verdade de uma proposição pode ser um subconjunto fuzzy de qualquer conjunto parcialmente ordenado (GOMIDE et al., 1995).

De acordo com Rodrigues et al. (2016), a Lógica Fuzzy é uma técnica que se adequa bem em situações onde não existe uma definição única de determinadas características, como é o caso da modelagem dos estilos de aprendizagem. 
Rodrigues et al. (2016) propõe uma abordagem baseada em Lógica Fuzzy para detecção dos estilos de aprendizagem dos estudantes. Nesse modelo, o autor classifica as notas do estudante de acordo com alguns conjuntos difusos: Muito Ruim, Ruim, Média, Boa e Muito Boa. Cada nota pertence a um determinado conjunto difuso com certo grau de pertinência que varia entre 0 e 1 (RODRIGUES et al., 2016).

Sendo assim, o sistema seleciona, por meio de algumas regras de defuzzificação, uma Combinação de Estilo de Aprendizagem que contenha uma baixa média de notas ruins e alta média de notas boas (RODRIGUES et al., 2016).

Os resultados do algoritmo foram comparados com resultados obtidos pelo algoritmo descrito por Dorça (2012). A abordagem proposta se mostrou superior devido à sua baixa variabilidade nos resultados e um melhor desempenho do algoritmo (RODRIGUES et al., 2016).

\subsection{Modelos Ocultos de Markov}

Um Modelo Oculto de Markov é uma variação das cadeias de Markov, pois além de apresentar as distribuições de probabilidades comuns às cadeias de Markov, ele possui também uma distribuição baseada em observação e comportamento do ambiente em que está inserido (RABINER, 1989).

De acordo com Rabiner (1989), o Modelo Oculto de Markov é um processo duplamente estocástico, onde um dos processos estocásticos é não visível e não observável, e o outro é um processo que produz uma sequência de observações. Dessa forma, o primeiro processo estocástico, não visível, pode ser observado pelo segundo processo estocástico, que produz a sequência de observações.

Alguns autores já utilizaram a abordagem de Modelo Oculto de Markov para detectar os estilos de aprendizagem de estudantes. Pode-se destacar as propostas de Nguyen (2013), Sena et al. (2016) e Almeida et al. (2019).

A abordagem proposta por Nguyen (2013) utiliza um Modelo Oculto de Markov para cada dimensão do FSLSM. As subdimensões das dimensões formam os estados ocultos do modelo, enquanto as observações das ações do estudante no sistema compõem os estados observados do modelo.

A inferência dos estilos de aprendizagem, no caso da modelagem por meio dos Modelos Ocultos de Markov, está relacionada a resolver o problema de encontrar a melhor sequência de estados ocultos que influenciou na geração dos estados observados do modelo. Ou seja, qual sequência de transição de estados é mais provável que tenha conduzido a uma sequência de observações (NGUYEN, 2013). A melhor solução para esse problema, conforme Nguyen (2013), é utilizar o Algoritmo de Viterbi.

Sena et al. (2016) implementou a modelagem de estilos de aprendizagem utilizando os Modelos Ocultos de Markov e o Algoritmo de Viterbi para inferir os estilos. Os resultados foram muito satisfatórios, com uma taxa de acerto de estilo de aprendizagem probabilístico acima de $85 \%$ para cada dimensão do FSLSM. 
Já em Almeida et al. (2019) foi implementada uma modelagem de estilos de aprendizagem utilizando Modelos Ocultos de Markov e uma abordagem de Aprendizagem por Reforço. Os resultados obtidos foram satisfatórios, com uma taxa de acerto superior a $91 \%$.

\subsection{Outras Técnicas para Detecção de Estilos de Aprendizagem}

Em Oliveira et al. (2018), é apresentado um modelo para detecção automática de estilos de aprendizagem que se baseia na meta-heurística Vitis Vinífera (MHV). A MHV é baseada no comportamento e crescimento da videira. Os autores compararam o desempenho da abordagem proposta com o Algoritmo Genético apresentado em Yannibelli et al. (2006). Os resultados apontam diferença significativa entre o MHV e o Algoritmo Genético, sendo que o MHV apresentou resultados melhores.

Em Ribeiro et al. (2017), é apresentada uma abordagem baseada em Dorça (2012) e que propõe o uso do conceito de Média Móvel Exponencialmente Ponderada (MMEP) com o objetivo de valorizar bons resultados encontrados em sessões de aprendizagem anteriores e acelerar 0 processo de detecção do estilo de aprendizagem do estudante. Os resultados do uso da MMEP apontam que o tempo médio para identificar as preferências do estudante, bem como o número de sessões de aprendizagem, foi menor do que a abordagem apresentada em Dorça (2012).

No trabalho proposto por Dung e Florea (2012), uma abordagem baseada em regras de mapeamento é utilizada. As regras de mapeamento são baseadas no tempo gasto pelos estudantes e no número de visitas a determinado objeto de aprendizagem. Para validação da proposta, foi utilizada uma base de dados com 44 estudantes de um curso de 9 meses de inteligência artificial. Os resultados obtidos pela abordagem foram comparados com o resultado obtido pelo questionário ILS, aplicado no início do curso. Para a dimensão Processamento, foi obtida uma média de $72,73 \%$ de inferências corretas; para a dimensão Percepção, 70,15\%; para a dimensão Entrada, 79,54\%; para a dimensão Organização, 65,91\%.

\section{Análise da Revisão}

A presente revisão bibliográfica realizou o levantamento de 26 trabalhos sobre detecção de estilos de aprendizagem. Além dos trabalhos clássicos sobre detecção de estilos de aprendizagem, foram considerados também trabalhos importantes desenvolvidos por pesquisadores brasileiros (as) e publicados em eventos e periódicos nacionais. A Figura 1 apresenta uma linha do tempo com as publicações relacionadas a detecção de estilos de aprendizagem exploradas na presente revisão.

A linha do tempo apresenta o trabalho e ano de publicação, bem como o método utilizado para realizar a modelagem e detecção do estilo de aprendizagem. Os métodos foram abreviados de acordo com a seguinte legenda: RB - Redes Bayesianas; ILS - Index of Learning Styles; RNA - Redes Neurais Artificiais; AD - Árvore de Decisão; AG - Algoritmo Genético; LSI - Learning Style Inventory; CM - Cadeias de Markov; AR - Aprendizagem por Reforço; HMM Modelos Ocultos de Markov; SVM - Máquina de Vetor de Suporte; MD - Mineração de Dados; MMEP - Média Móvel Exponencialmente Ponderada; DS - Dynamic Scripting; MHV - Metaheurística Vitis Vinífera.

Conforme pode ser visualizado na Figura 1, existem diversas abordagens para detecção dos 
estilos de aprendizagem. Ao todo, foram levantados 15 métodos distintos, sendo que alguns deles utilizam mais de uma técnica para realizar a modelagem e detecção dos estilos de aprendizagem.

Figura 1 - Linha do tempo dos trabalhos relacionados a detecção de estilos de aprendizagem

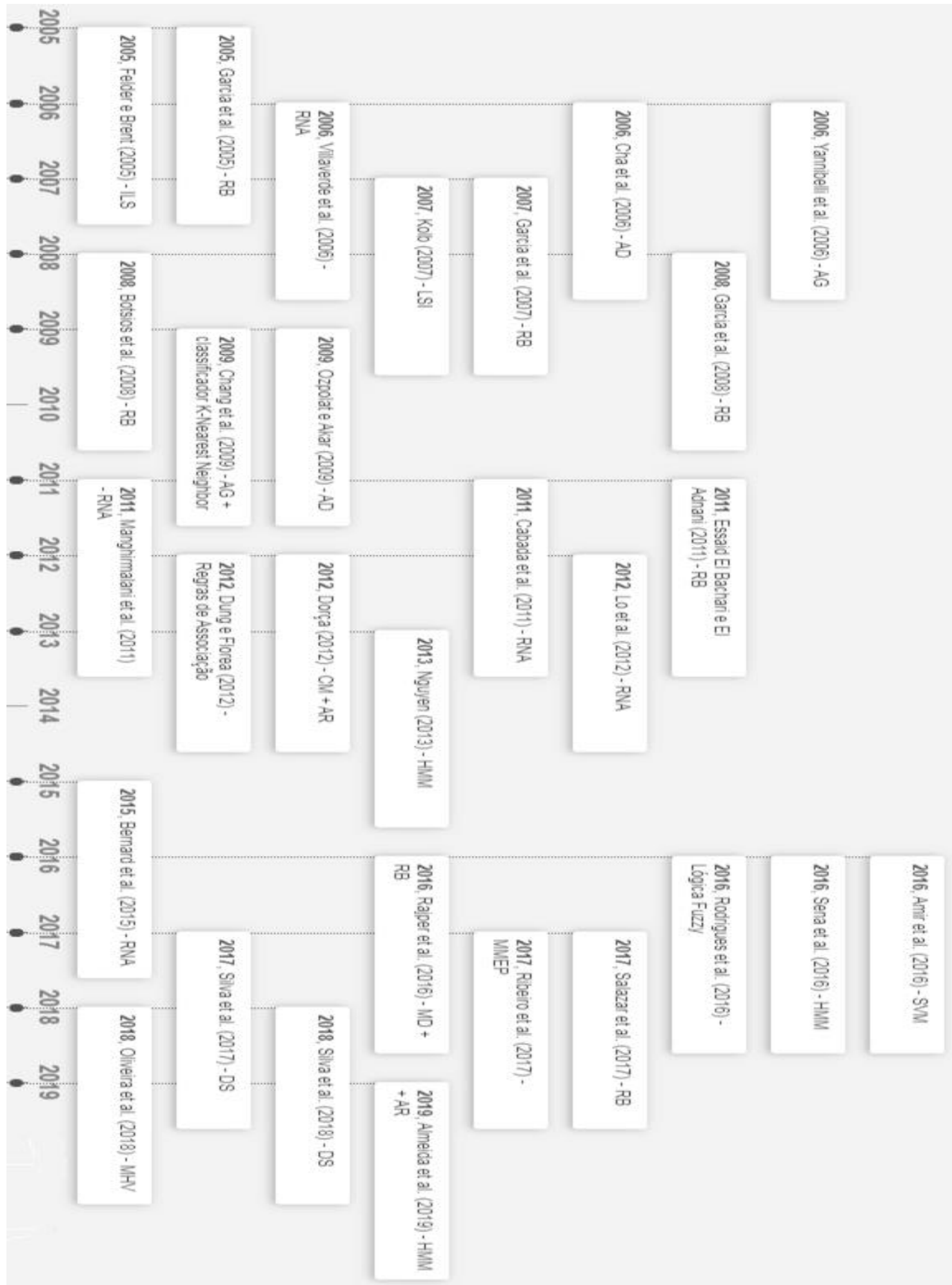


No trabalho de Kirshner (2017), o autor faz críticas aos estilos de aprendizagem. Segundo o autor, a teoria dos estilos de aprendizagem é uma crença que apresenta pouca evidência científica, pouca base teórica e ausência de evidência empírica significativa (KIRSHNER, 2017). O autor recomenda ainda que os pesquisadores parem de propagar a teoria, considerada por ele como um mito.

Com as críticas apresentadas em Kirshner (2017), apenas dois anos depois os estilos de aprendizagem ainda parecem ser objeto de estudo de alguns pesquisadores. Conforme linha do tempo apresentada na Figura 1, foram 4 trabalhos publicados em 2016 (anteriores ao trabalho do Kirshner), 3 trabalhos publicados em 2017 (ano do trabalho do Kirshner), 2 em 2018 e 1 em 2019. A quantidade de trabalhos nesses anos é absolutamente compatível com o de outros anos, como 2011 (3 trabalhos), 2012 (3 trabalhos) e 2013 (1 trabalho).

Dessa forma, não se pode dizer que após o trabalho de Kirshner (2017) e as críticas que ele apresenta as pesquisas sobre estilos de aprendizagem estejam em declínio. Com as diversas abordagens automáticas para detecção dos estilos de aprendizagem, como as apresentadas neste trabalho, as pesquisas na área ainda devem obter avanços consideráveis e auxiliar na adaptação dos Ambientes Virtuais de Aprendizagem.

A Tabela 1 apresenta uma síntese dos trabalhos e das técnicas computacionais para detecção de EA. Pela tabela é possível perceber que o modelo mais utilizado para descrição dos EAs em ambientes computacionais é o FSLSM. Ele está presente em 21 dos 26 trabalhos. Isso se deve principalmente a: sua simplicidade de representação, visto que o modelo possui 4 dimensões com 2 subdimensões cada, totalizando 16 combinações possíveis de EA, sendo simples sua representação computacional; sua característica de não ser estático, refutando assim uma crítica a teoria de EA; e também por ser um modelo já consolidado.

Tabela 1 - Trabalhos e técnicas para detecção automática de EA

\begin{tabular}{|c|c|c|}
\hline $\begin{array}{c}\text { Abordagem } \\
\text { Utilizada }\end{array}$ & Trabalhos Relacionados & Modelo de EA Utilizado \\
\hline Redes Bayesianas & $\begin{array}{c}\text { Garcia et al. (2005), Garcia et al. (2007), } \\
\text { Garcia et al. (2008), Botsios et al. (2008), } \\
\text { Essaid; El Bachari; El Adnani (2011) e } \\
\text { Salazar et al. (2017). }\end{array}$ & $\begin{array}{l}\text { - Garcia et al. (2005, 2007, 2008): FSLSM; } \\
\text { - Botsios et al. (2008): KOLB; } \\
\text { - Essaid; El Bachari; El Adnani (2011): MBTI; } \\
\text { - Salazar et al. (2017): FSLSM. }\end{array}$ \\
\hline $\begin{array}{l}\text { Redes Neurais } \\
\text { Artificiais }\end{array}$ & $\begin{array}{l}\text { Villaverde et al. (2006), Cabada et al. } \\
\text { (2011), Lo et al. (2012) e Bernard et al. } \\
\text { (2015). }\end{array}$ & $\begin{array}{l}\text { - Villaverde et al. (2006): FSLSM; } \\
\text { - Cabada et al. (2011): FSLSM; } \\
\text { - Lo et al. (2012): MBTI; } \\
\text { - Bernard et al. (2015): FSLSM. }\end{array}$ \\
\hline Árvore de Decisão & Cha et al. (2006) e Ozpolat e Akar (2009). & $\begin{array}{l}\text { - Cha et al. (2006): FSLSM; } \\
\text { - Ozpolat e Akar (2009): FSLSM. }\end{array}$ \\
\hline $\begin{array}{l}\text { Algoritmo Genético } \\
+ \text { K Nearest } \\
\text { Neighbor }\end{array}$ & Chang et al. (2009). & Não utiliza um modelo específico de EA. \\
\hline Algoritmo Genético & Yannibelli et al. (2006). & FSLSM \\
\hline $\begin{array}{c}\text { Aprendizagem por } \\
\text { Reforço e Cadeia } \\
\text { de Markov }\end{array}$ & Dorça (2012). & FSLSM \\
\hline Dynamic Scripting & Silva et al. (2017) e Silva et al. (2018). & FSLSM \\
\hline $\begin{array}{l}\text { Mineração de } \\
\text { Dados } \\
\text { Educacionais } \\
\end{array}$ & Rajper et al. (2016). & KOLB \\
\hline Lógica Fuzzy & Rodrigues et al. (2016). & FSLSM \\
\hline $\begin{array}{c}\text { Modelos Ocultos de } \\
\text { Markov }\end{array}$ & Nguyen (2013), Sena et al. (2016). & FSLSM \\
\hline $\begin{array}{l}\text { Modelos Ocultos de } \\
\text { Markov }+ \\
\text { Aprendizagem por } \\
\text { Reforço } \\
\end{array}$ & Almeida et al. (2019) & FSLSM \\
\hline $\begin{array}{c}\text { Meta-heurística } \\
\text { Vitis Vinífera } \\
\end{array}$ & Oliveira et al. (2018). & FSLSM \\
\hline
\end{tabular}




\begin{tabular}{|c|c|c|}
\hline (MHV) & Ribeiro et al. (2017). & FSLSM \\
\hline $\begin{array}{c}\text { Expódia Móvel } \\
\text { Ponencialmente }\end{array}$ & Dung e Florea (2012). \\
$\begin{array}{c}\text { Regras de } \\
\text { Mapeamento }\end{array}$ & Amir et al. (2016). & FSLSM \\
\hline $\begin{array}{c}\text { Máquina de Vetor } \\
\text { de Suporte (SVM) }\end{array}$ & FSLSM \\
\hline
\end{tabular}

Fonte: Próprio autor.

\section{Considerações Finais}

A partir dos fundamentos abordados neste trabalho, percebe-se que os estilos de aprendizagem e suas formas de detecção são um fator determinante para a criação de ambientes computacionais que se adaptem ao estudante, oferecendo melhorias ao processo de ensino-aprendizagem. Dessa forma, este é um amplo horizonte a ser explorado na EaD e, por isso, este é um tópico que vem sendo constantemente objeto de estudo na área da Informática aplicada a Educação.

Existem na literatura diversas abordagens para deteç̧ão de estilos de aprendizagem de estudantes em Sistemas Educacionais. Desde abordagens colaborativas, que utilizam questionários para levantamento das preferências dos estudantes, até abordagens automáticas, que utilizam técnicas avançadas de Inteligência Artificial, Inteligência Computacional e Aprendizado de Máquina para detectar os estilos de aprendizagem.

Na pesquisa bibliográfica realizada nesse trabalho, a abordagem para deteç̧ão automática de estilos de aprendizagem mais utilizada na literatura é por meio de Redes Bayesianas, com 6 trabalhos encontrados. Em segundo, a técnica de Redes Neurais, com 4 trabalhos relacionados. As outras abordagens descritas nesse artigo têm 3, 2 ou 1 trabalho relacionado. Por possuírem mais trabalhos relacionados e também por serem amplamente utilizadas na área da computação, as abordagens que utilizam Redes Bayesianas e Redes Neurais Artificiais são promissoras para serem implementadas em um ambiente virtual de aprendizagem real.

O presente trabalho cumpre, assim, o seu propósito inicial de realizar uma revisão e descrição sobre as diversas técnicas presentes na literatura para identificação dos estilos de aprendizagem dos estudantes em Sistemas para Educação à Distância.

\section{Referências}

AL-AZAWEI, A.; BADII, A. State of the art of learning styles-based adaptive educational hypermedia systems (LS-BAEHSs). International Journal of Computer Science \& Information Technology, Academy \& Industry Research Collaboration Center (AIRCC), v. 6, n. 3, p. 1, 2014.

ALMEIDA, Arthur Machado França de et al. Utilização de Modelos Ocultos de Markov e Aprendizagem por Reforço para detecção de Estilos de Aprendizagem de estudantes em Ambientes Virtuais de Ensino e Aprendizagem. Revista Eletrônica Argentina-Brasil de Tecnologias da Informação e da Comunicação, [S.I.], v. 1, n. 10, jun. 2019.

AMIR, E. S.; SUMADYO, M.; SENSUSE, D. I.; SUCAHYO, Y. G.; SANTOSO, H. B. Automatic detection of learning styles in learning management system by using literature-based method and support vector machine. In: IEEE. Advanced Computer Science and Information Systems (ICACSIS), 2016 International Conference on. [S.I.], 2016.

AVGERIOU, P.; PAPASALOUROS, A.; RETALIS, S.; SKORDALAKIS, M. Towards a pattern language for learning management systems. Educational Technology \& Society, JSTOR, v. 6, n. 2, p. 11-24, 2003. 
BAKER, R.; ISOTANI, S.; CARVALHO, A. Mineração de dados educacionais: Oportunidades para o brasil. Brazilian Journal of Computers in Education, v. 19, n. 02, p. 3, 2011.

BARROS, D. M. V.; GARCÍA, C. A.; DO AMARAL, S. F. Estilo de uso do espaço virtual. Journal of Learning Styles, v. 1, n. 1, 2008.

BITTENCOURT, I. M.; MERCADO, L. P. L. Evasão nos cursos na modalidade de educação a distância: estudo de caso do curso piloto de administração da ufal/uab. Revista Ensaio: Avaliação e Políticas Públicas em Educação, v. 22, n. 83, p. 465-504, 2014.

BOTSIOS, S.; GEORGIOU, D.; SAFOURIS, N. Contributions to adaptive educational hypermedia systems via on-line learning style estimation. Educational Technology \& Society, JSTOR, v. 11, n. 2, p. 322-339, 2008.

BERNARD, J.; CHANG, T. W.; POPESCU, E.; GRAF, S. Using artificial neural networks to identify learning styles. International Conference on Artificial Intelligence in Education. Springer, Cham, 2015.

BRASIL. Lei de Diretrizes e Bases da educação Nacional. Diario Oficial da União, Brasília, Seção 1, p. 27839, 1996. Disponível em: <http://www.planalto.gov.br/ccivil 03/leis/L9394.htm>.

BROWN, E., BRAILSFORD, T., FISHER, T., MOORE, A., and ASHMAN, H. Reappraising cognitive styles in adaptive web applications. In Proceedings of the 15th international conference on World Wide Web, pages 327-335, 2006.

BRUSILOVSKY, P. Methods and techniques of adaptive hypermedia. User modeling and user-adapted interaction, Springer, v. 6, n. 2-3, p. 87-129, 1996.

CABADA, R. Z.; ESTRADA, M. L. B.; GARCIA, C. A. R. EDUCA: A web 2.0 authoring tool for developing adaptive and intelligent tutoring systems using a Kohonen network. Expert Systems with Applications, Elsevier, v. 38, n. 8, p. 9522-9529, 2011.

CHA, H. J.; KIM, Y. S.; PARK, S. H.; YOON, T. B.; JUNG, Y. M.; LEE, J.-H. Learning styles diagnosis based on user interface behaviors for the customization of learning interfaces in an intelligent tutoring system. In: SPRINGER. International Conference on Intelligent Tutoring Systems. [S.I.], 2006. p. 513-524.

CHANG, Y.-C.; KAO, W.-Y.; CHU, C.-P.; CHIU, C.-H. A learning style classification mechanism for e-learning. Computers \& Education, Elsevier, v. 53, n. 2, p. 273-285, 2009.

DORÇA, F. A. Uma abordagem estocástica baseada em Aprendizagem por Reforço para modelagem automática e dinâmica de Estilos de Aprendizagem de Estudantes em Sistemas Adaptativos e Inteligentes para Educação a Distância. Tese (Doutorado), 2012.

DUNG, P. Q.; FLOREA, A. M. An approach for detecting learning styles in learning management systems based on learners' behaviours. In International Conference on Education and Management Innovation, $v$. 30, p. 171-177, 2012.

Essaid El Bachari, E. H. A.; El Adnani, M. E-LEARNING PERSONALIZATION BASED ON DYNAMIC LEARNERS'PREFERENCE. Citeseer, 2011.

FELDER, R. M.; BRENT, R. Understanding student differences. Journal of engineering education, Wiley Online Library, v. 94, n. 1, p. 57-72, 2005.

FELDER, R. M.; SILVERMAN, L. K. et al. Learning and teaching styles in engineering education. Engineering education, v. 78, n. 7, p. 674-681, 1988.

FELDER, R. M.; SPURLIN, J. Applications, reliability and validity of the index of learning styles. International journal of engineering education, v. 21, n. 1, p. 103-112, 2005.

GARCIA, P.; AMANDI, A.; SCHIAFFINO, S.; CAMPO, M. Using Bayesian networks to detect students' learning styles in a web-based education system. Proc of ASAI, Rosario, p. 115-126, 2005. 
GARCIA, P.; AMANDI, A.; SCHIAFFINO, S.; CAMPO, M. Evaluating Bayesian networks precision for detecting students learning styles. Computers \& Education, Elsevier, v. 49, n. 3, p. 794-808, 2007.

GARCIA, P.; SCHIAFFINO, S.; AMANDI, A. An enhanced Bayesian model to detect students' learning styles in Web-based courses. Journal of Computer Assisted Learning, Wiley Online Library, v. 24, n. 4, p. 305$315,2008$.

GOMIDE, F.; GUDWIN, R. R.; TANSCHEIT, R. Conceitos fundamentais da teoria de conjuntos fuzzy, lógica fuzzy e aplicações. In: Proc. 6 th IFSA Congress-Tutorials. [S.I.: s.n.], 1995. p. 1-38.

GONG, W.; WANG, W. Application research of support vector machine in e-learning for personality. In: IEEE. Cloud Computing and Intelligence Systems (CCIS), 2011 IEEE International Conference on. [S.I.], 2011. p. 638-642.

GRAF, S.; IVES, C. et al. A flexible mechanism for providing adaptivity based on learning styles in learning management systems. In: IEEE. Advanced Learning Technologies (ICALT), 2010 IEEE 10th International Conference on. [S.I.], 2010. p. 30-34.

GRAF, S.; LIN, T. et al. Analysing the relationship between learning styles and cognitive traits. In: IEEE. Advanced Learning Technologies, 2007. ICALT 2007. Seventh IEEE International Conference on. [S.I.], 2007. p. 235-239.

HONEY, P.; MUMFORD, A. The manual of learning styles. Peter Honey Publications, 1992.

KIRSCHNER, P. A. Stop propagating the learning styles myth. Computers \& Education, Elsevier, v. 106, p. 166-171, 2017.

KIRSCHNER, P. A.; MERRIËNBOER, J. J. van. Do learners really know best? urban legends in education. Educational psychologist, Taylor \& Francis, v. 48, n. 3, p. 169-183, 2013.

KOLB, D. A.; BOYATZIS, R. E.; MAINEMELIS, C. Experimental Learning: Experience as the source of learning and development. [S.I.]: Prentice Hall Englewood, 1984.

LO, J.-J.; CHAN, Y.-C.; YEH, S.-W. Designing an adaptive web-based learning system based on students' cognitive styles identified online. Computers \& Education, Elsevier, v. 58, n. 1, p. 209-222, 2012.

MIRANDA, Luísa et al. Estilos de aprendizagem: uso do virtual pelos estudantes do ensino superior. Learning Style Review, p. 184-195, 2012.

MOORE, M. G.; KEARSLEY, G. Distance education: A systems view of online learning. [S.I.]: Cengage Learning, 2011.

NANCEKIVELL, S. E.; SHAH, P.; GELMAN, S. A. Maybe they're born with it, or maybe it's experience: Toward a deeper understanding of the learning style myth. Journal of Educational Psychology, 2019.

NETO, W. C. B. Web semântica na construção de sistemas de aprendizagem adaptativos. Tese (Doutorado), 2006.

NETTO, H. G. C.; SOUZA, R. N. P. M. A importância da educação a distância na formação do conhecimento para os concursos públicos. Brazilian Journal of Development, v. 5, n. 6, p. 4590-4598, 2019.

NGUYEN, L. A new approach for modeling and discovering learning styles by using hidden markov model. Global Journal of Human-Social Science Research, v. 13, n. 4, 2013.

OZPOLAT, E.; AKAR, G. B. Automatic detection of learning styles for an e-learning system. Computers \& Education, Elsevier, v. 53, n. 2, p. 355-367, 2009.

PASHLER, H.; MCDANIEL, M.; ROHRER, D.; BJORK, R. Learning styles: Concepts and evidence. Psychological science in the public interest, SAGE Publications Sage CA: Los Angeles, CA, v. 9, n. 3, p. 105119, 2008. 
PASK, G. Styles and strategies of learning. British journal of educational psychology, Wiley Online Library, $v$. 46 , n. 2, p. $128-148,1976$.

PUGA, S. G. Sistemas Hipermídia adaptativos para a educação baseada na web: uma visão semiótica. Tese (Doutorado) - Universidade de São Paulo, 2008.

RABIN, S. AI Game Programming Wisdom. Charles River Media; Boston, 2002.

RABINER, L. R. A tutorial on hidden markov models and selected applications in speech recognition. Proceedings of the IEEE, Ieee, v. 77, n. 2, p. 257-286, 1989.

RAJPER, S.; SHAIKH, N. A.; SHAIKH, Z. A.; MALLAH, G. A. Automatic detection of learning styles on learning management systems using data mining technique. Indian Journal of Science and Technology, v. 9, n. 15, 2016.

RAMOS, D. Cursos on-line: planejamento e organização. Florianópolis: Ed. da UFSC, 2010.

RAWSON, K.; STAHOVICH, T. F.; MAYER, R. E. Homework and achievement: Using smartpen technology to find the connection. Journal of Educational Psychology, American Psychological Association, v. 109, n. 2, p. $208,2017$.

REID, J. M. Understanding learning styles in the second language classroom. [S.I.]: Prentice Hall Regents, 1998.

RIBEIRO, P.; ASSIS, L.; VIVAS, A.; PITANGUI, C. Detecção de estilos de aprendizagem utilizando média móvel exponencialmente ponderada. In: Brazilian Symposium on Computers in Education (Simpósio Brasileiro de Informática na Educação-SBIE). [S.I.: s.n.], 2017. v. 28, n. 1, p. 1247.

RODRIGUES, L. H. S.; VIVAS, A.; ASSIS, L.; PITANGUI, C.; FALCI, S. Uso de lógica fuzzy na seleção de estratégias de aprendizagem. XXVII Simpósio Brasileiro de Informática na Educação, 2016.

RUSSELL, S.; NORVIG, P. Artificial intelligence: a modern approach. Prentice-Hall, Egnlewood Cliffs, v. 25, 1995

SALAZAR, L.; ASSIS, L.; VIVAS, A.; PITANGUI, C.; FALCI, S. Detecção de estilos de aprendizagem em ambientes virtuais de aprendizagem utilizando redes bayesianas. In: Brazilian Symposium on Computers in Education (Simpósio Brasileiro de Informática na Educação-SBIE). [S.I.: s.n.], 2017. v. 28, n. 1, p. 1247.

SENA, E.; VIVAS, A.; ASSIS, L.; PITANGUI, C. Proposta de uma abordagem computacional para detecção automática de estilos de aprendizagem utilizando modelos ocultos de markov e fs/sm. In: Brazilian Symposium on Computers in Education (Simpósio Brasileiro de Informática na Educação-SBIE). [S.I.: s.n.], 2016. v. 27 , n. 1 , p. 1126.

SENECHAL, A. C. L. Analise e pré-processamento de dados utilizando técnicas de mineração de dados educacionais para o Moodle. 2013.

SILVA, D. M. d. O impacto dos estilos de aprendizagem no ensino de contabilidade na FEA-RP/USP. Tese (Doutorado) - Universidade de Sao Paulo, 2006.

SILVA, E. V. d. Educação a distância: uma realidade na formação docente inicial. Brazilian Journal of Development, v. 5, n. 7, p. 9854-9866, 2019.

SILVA, J. C. C.; PITANGUI, C. G.; ASSIS, L; VIVAS, A. Detecção Automática e Dinâmica de Estilos de Aprendizagem em Sistemas Adaptativos e Inteligentes utilizando Dynamic Scripting. In: Brazilian Symposium on Computers in Education (Simpósio Brasileiro de Informática na Educação-SBIE). [S.I.: s.n.], 2017. p. 1327

SILVA, J. C. C.; PITANGUI, C. G.; VIVAS, A.; ASSIS, L. Uma Nova Abordagem para Sistemas Adaptativos e Inteligentes utilizando Dynamic Scripting. Revista Eletrônica Argentina-Brasil de Tecnologias da Informação e da Comunicação, [S.I.], v. 1, n. 8, abr. 2018. ISSN 2446-7634. Disponível em: <https://revistas.setrem.com.br/index.php/reabtic/article/view/271>. Acesso em: 14 jul. 2019. 
SILVA, L. L. V. Estilos e estratégias de aprendizagem de estudantes universitários. Tese (Doutorado) Universidade de Sao Paulo, 2012.

SOARES, C. S. A.; SOARES, P. H. A. A IMPORTÂNCIA DA UTILIZAÇÃO DE VARIADOS ESTILOS DE APRENDIZAGEM NO ENSINO SUPERIOR. Amazônica-Revista de Psicopedagogia, Psicologia escolar e Educação, v. 23, n. 2, p. 338-356, 2019.

SPRONCK, P. H. M. Adaptive game AI. Tese (Doutorado) - Maastricht University, 2005.

VEENMAN, M. V.; PRINS, F. J.; VERHEIJ, J. Learning styles: Self-reports versus thinking-aloud measures. British Journal of Educational Psychology, Wiley Online Library, v. 73, n. 3, p. 357-372, 2003.

VILLAVERDE, J. E.; GODOY, D.; AMANDI, A. Learning styles' recognition in e-learning environments with feed-forward neural networks. Journal of Computer Assisted Learning, Wiley Online Library, v. 22, n. 3, p. 197-206, 2006.

YANNIBELLI, V.; GODOY, D.; AMANDI, A.A genetic algorithm approach to recognise students' learning styles. Interactive Learning Environments, Informa UK Limited, v. 14, n. 1, p. 55-78, 2006.

ZAPALSKA, A. and BROZIK, D. Learning styles and online education. Campus-Wide Information Systems, p, 325-335, 2006.

Recebido em outubro de 2019.

Aprovado para publicação em fevereiro de 2020.

\section{Arthur Machado França de Almeida}

Coordenadoria de Gestão de TI - Instituto Federal do Norte de Minas Gerais - IFNMG, Campus Araçuaí, Brasil, arthurmfalmeida@gmail.com.

\section{Luciana Pereira de Assis}

Departamento de Computação - Universidade Federal dos Vales do Jequitinhonha e Mucuri - UFVJM, Campus JK, Brasil, lupassis@gmail.com.

\section{Alessandro Vivas Andrade}

Departamento de Computação - Universidade Federal dos Vales do Jequitinhonha e Mucuri - UFVJM, Campus JK, Brasil, alessandro.vivas@gmail.com. 